\title{
IMPORTÂNCIA E DESAFIOS FRENTE À IMPLANTAÇÃO DO PROTOCOLO DE CIRURGIA SEGURA
}

\author{
IMPORTANCE AND CHALLENGES IN FRONT OF THE IMPLEMENTATION OF THE SAFE SURGERY \\ PROTOCOL
}

\section{IMPORTANCIA Y DESAFÍOS FRENTE A LA IMPLEMENTACIÓN DEL PROTOCOLO DE CIRUGÍA SEGURA}

\author{
Ivonete Lopes ${ }^{1}$, Raphael Alves da Silva ${ }^{2}$,Henry Johnson Passos de Oliveira ${ }^{3}$, Elizandra Cássia da Silva \\ Oliveira ${ }^{4}$, Emanuela Batista Ferreira e Pereira ${ }^{5}$, Felicialle Pereira da Silva ${ }^{6}$
}

Submetido: $10 / 08 / 2020$

Aprovado: 25/08/2020

\section{RESUMO}

Introdução: as complicações cirúrgicas mobilizam preocupação sobre riscos inerentes ao procedimento cirúrgico, podendo levar a incapacidades irreversíveis, ou até a morte do paciente. Objetivo: Identificar a importância da aplicação do checklist da cirurgia segura. Método: revisão integrativa da literatura, nas bases LILACS, MEDLINE e BDENF realizada de junho a julho de 2020. Utilizando as palavras chaves: cirurgia segura; centro cirúrgico; enfermagem. Buscaram-se artigos publicados em português, inglês e espanhol, sem limite de período. Resultados: a amostra final foi constituída de 10 artigos que evidenciaram categorias: estabelecimento da comunicação e interação entre a equipe; cuidado seguro para redução do medo e ansiedade e conhecimento do protocolo de cirurgia segura salva vidas. Discussão: a comunicação é essencial nas interações humanas, quando efetiva pode reduzir erros evitáveis para o paciente. O cheklist deve ser uma construção diária na assistência. Conclusão: apesar da preocupação com segurança do paciente, a implementação de metas internacionais ainda é um desafio para adesão à uma cultura de segurança.

PALAVRAS-CHAVE: Procedimentos Cirúrgicos Ambulatórios; Centros Cirúrgicos; Cuidados de Enfermagem. to irreversible disabilities, or even the death of the patient. Objective: To identify the importance of applying the safe surgery checklist. Method: integrative literature review, based on LILACS, MEDLINE and BDENF carried out between from June to July 2020. Using the keywords: safe surgery; surgery Center; nursing. Articles published in Portuguese, English and Spanish were searched, with no time limit. Results: the final sample consisted of 10 articles that showed categories: establishment of communication and interaction between the team; safe care to reduce fear and anxiety and knowledge of the safe surgery protocol saves lives. Discussion: communication is essential

${ }^{1}$ Enfermeira, especialista em Centro Cirúrgico. Faculdade IDE, Departamento de Enfermagem. Recife, Pernambuco, Brasil. https://orcid.org/0000-0003-1136-7230

${ }^{2}$ Acadêmico de Enfermagem. Centro Universitário Mauricio de Nassau- UNINASSAU, Departamento de Enfermagem. RecifePE. https://orcid.org/0000-0003-2555-909X, raphaelalves770@hotmail.com.

${ }^{3}$ Enfermeiro, Residente em Saúde Coletiva pelo Instituto Aggeu Magalhães- FIOCRUZ-PE, departamento de saúde coletiva. Recife-PEI.

${ }^{4}$ Enfermeira doutora em enfermagem Universidade de Pernambuco- UPE-FENSG, Departamento de Enfermagem. Recife-PE ${ }^{5}$ Enfermeira doutora em enfermagem, professora adjunta da Universidade de Pernambuco- UPE-FENSG, Departamento de Enfermagem. Recife-PE

${ }^{6}$ Enfermeira Doutora em Neuropsiquiatria e ciências do comportamento- UFPE, professora do curso de enfermagem do Centro Universitário Mauricio de Nassau- UNINASSAU e professora adjunta da Universidade de Pernambuco- UPE,
} 
in human interactions, when effective it can reduce avoidable errors for the patient. The checklist should be a daily build in service. Conclusion: despite the concern for patient safety, the implementation of international goals is still a challenge to adhere to a safety culture.

KEYWORDS: Ambulatory Surgical Procedures; Surgical Centers; Nursing Care.

\section{RESUMEN}

Introducción: las complicaciones quirúrgicas plantean inquietudes sobre los riesgos inherentes al procedimiento quirúrgico, que pueden conducir a discapacidades irreversibles o incluso a la muerte del paciente. Objetivo: Identificar la importancia de aplicar el checklist de cirugía segura. Método: revisión integradora de la literatura, basada en LILACS, MEDLINE y BDENF llevado a cabo entre junio a julio de 2020. Utilizando las palabras clave: cirugía segura; centro cirúrgico; enfermería. Se realizaron búsquedas de artículos publicados en portugués, inglés y español, sin límite de tiempo. Resultados: la muestra final estuvo conformada por 10 artículos que presentaban categorías: establecimiento de comunicación e interacción entre el equipo; la atención segura para reducir el miedo y la ansiedad y el conocimiento del protocolo de cirugía segura salva vidas. Discusión: la comunicación es esencial en las interacciones humanas, cuando es efectiva puede reducir errores evitables para el paciente. La lista de verificación debe ser un servicio incorporado diario. Conclusión: a pesar de la preocupación por la seguridad del paciente, la implementación de los objetivos internacionales sigue siendo un desafío para adherirse a una cultura de seguridad.

PALABRAS CLAVE: Procedimientos quirúrgicos ambulatorios; Centros quirúrgicos; Atención de Enfermería. 


\section{INTRODUÇÃO}

Os avanços tecnológicos e o aumento da qualidade da assistência hospitalar possibilitaram o aumento no número de procedimentos cirúrgicos. Desta forma, os danos relacionados à assistência foram evidenciados. A publicação do relatório americano intitulado "To Err is Human", proporcionou amplas discussões no âmbito científico, revelando que cerca de 44.000 a 98.000 pessoas morriam todos os anos nos Estados Unidos da América devido aos erros na assistência médico-hospitalar 1-2

As complicações cirúrgicas neste sentido mobilizam preocupações a respeito dos riscos que são considerados inerentes ao procedimento cirúrgico, podendo levar a incapacidades irreversíveis, ou até mesmo a morte do paciente. Neste contexto, as discussões sobre a segurança do paciente para redução do risco de danos desnecessários têm-se ampliado, contribuindo para que programas de segurança sejam implementados nas instituições assistenciais3.

No Brasil, o Programa Nacional de Segurança do Paciente foi instituído pelo Ministério da Saúde (MS) pela portaria 529/2013, recomendando aplicação sistêmica de políticas, procedimentos e condutas que resguardam o controle de riscos e de eventos adversos que afetam a segurança do paciente. A Resolução do Diretório Colegiada ( $R D C n^{\circ} 36$ de 25 de julho de 2013) é uma resolução que exige dos serviços de saúde, a criação dos Núcleos de Segurança do Paciente (NSP). Entre os protocolos a ser implantados, está o da cirurgia segura, que representa um desafio enfatizado pela campanha "Cirurgias Seguras Salvam Vidas"4-5.

A apresentação do Manual Cirurgia Segura Salvam Vidas no Brasil, foi resultado de uma parceria entre o Ministério da Saúde com a Organização Pan-americana e a Organização Mundial de Saúde (OMS), que visa colaborar com as práticas de prevenção dos riscos associados à assistência cirúrgica. Trata-se de um manual que apresenta uma lista de verificação de segurança cirúrgica (checklist), desenvolvido por especialistas, sendo dividido em três etapas: identificação (antes da indução anestésica), confirmação (antes da incisão cirúrgica) e registro (antes da saída do paciente da sala de cirurgia)2-6.

Pesquisa realizada em 2015 divulgou que houve redução nas complicações e mortalidade decorrentes de erros em cirurgia, após a implantação do checklist cirúrgico. Este instrumento reforça as práticas de segu- rança e redução de eventos adversos. A lista de verificação durante a cirurgia deverá ter uma única pessoa responsável, um coordenador, que frequentemente será um profissional de enfermagem, mas também pode ser qualquer outro profissional da saúde participante da cirurgia2-7.

A implementação do checklist é considerado de baixo custo, além disso, apresenta de forma reduzida todas as informações necessárias, tornando-se possível a sua aplicabilidade no processo de verificação, no tempo estimado de três minutos. Apesar dos benefícios verificados com a adoção do protocolo de segurança do paciente, a adesão ao seu cumprimento tornou-se um desafio, uma vez que na vivência prática os profissionais de saúde têm relatado barreiras para a adesão sistemática do checklist na sala de cirurgias.1-7

Neste sentido, compreende-se que não basta apenas que o checklist esteja implantado para garantir a adesão da equipe em sua rotina de trabalho. Entretanto, trata-se de uma necessidade vital para o cumprimento da segurança do paciente e para harmonia da equipe cirúrgica. Destarte, esta pesquisa se justifica pela imperiosa preocupação em identificar os aspectos que dificultam a aplicação da cirurgia segura e que se configura como desafio nas instituições de saúde.

Para a realização desta pesquisa, buscaram-se os pressupostos da pesquisa baseada em evidências que representa um processo de busca, avaliação e aplicação de evidências pela revisão integrativa da literatura por possibilitar a síntese e análise do conhecimento sobre o tema investigado8. O objetivo consiste em Identificar a importância da aplicação do checklist da cirurgia segura. Espera-se contribuir e somar esforços pela melhoria da assistência ao paciente.

\section{MÉTODO}

O método de síntese de conhecimento adotado foi a revisão integrativa. Para a condução desta investigação, percorreram-se cinco etapas: elaboração da questão de pesquisa (identificação do problema), busca na literatura dos estudos primários, avaliação dos estudos primários, análise dos dados e apresentação da revisão. A questão de pesquisa foi realizada com base na estratégia PICo(9), onde: P- População: pacientes cirúrgicos; I- Interesse: segurança do paciente; Co- Contexto: implementação do checklist, o que resultou na seguinte questão norteadora: "'Quais são as evidências disponíveis na literatura sobre os aspectos relevantes para apli- 
cabilidade do checklist, no centro cirúrgico? Ressalta-se que o elemento $C$, de comparação entre intervenção ou grupo, não foi empregado devido ao tipo de revisão.

Para a busca dos estudos primários, foram selecionadas as bases de dados Medical Literature Analysis and Retrieval Sistem on line (MEDLINE), Literatura Latino-americana e do Caribe em Ciências da Saúde (LILACS), e na Base de Dados de Enfermagem (BDENF). Para elaboração deste estudo, as etapas que guiam este método de pesquisa foram percorridas: estabelecimento dos objetivos, dos critérios de inclusão e exclusão, definição das informações a serem extraídas dos artigos selecionados, a análise dos resultados, discussão e apresentação dos resultados. Os critérios de seleção delimitados foram estudos primários que abordavam os aspectos sobre a implementação do checklist cirúrgico cujos autores investigaram conhecimento, estratégias relevantes e desafios sobre a implementação do checklist no centro cirúrgico.

Os critérios de inclusão dos artigos definidos inicialmente foram: artigos publicados em português, inglês e espanhol, com os resumos disponíveis nas bases de dados selecionados, nos últimos cinco anos. Palavras-chave: cirurgia segura, centro cirúrgico e enfermagem, pela busca integrada com o operador boleano AND. A busca foi realizada on line, entre os meses de junho e julho de 2020, utilizando-se os critérios de inclusão, a amostra final desta revisão foram 10 artigos. A delimitação desse período é justificada para assegurar quantitativo adequado de estudos primários.

Para a análise e posterior síntese dos artigos que atenderam aos critérios de inclusão foi utilizado um quadro sinóptico especialmente construído para esse fim, que contemplou os seguintes aspectos pertinentes: nome da pesquisa; nome dos autores; objetivos; resultados e conclusões. A apresentação dos resultados e discussão dos dados obtidos foi realizada de forma descritiva, com categorias elencadas para discussão, que possibilitam ao leitor pontos de partida para responder à questão de pesquisa e impactar positivamente na compreensão dos objetivos deste estudo10.

Ainda, para organizar o processo da amostra final dos artigos incluídos na revisão, foi realizado um fluxograma (Figura 1), que mostra de forma detalhada este processo e segue o modelo do Preferred Reporting Items for Systematic Reviewsand Meta-Analyses (PRISMA).
Figura 1 - Fluxograma utilizado para seleção dos artigos.

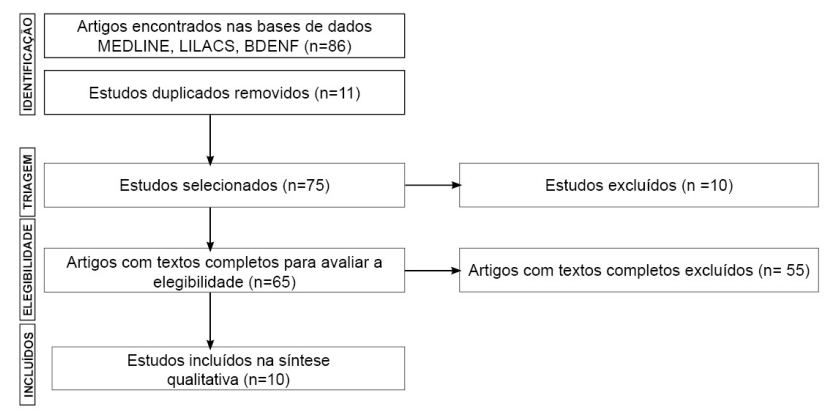

\section{RESULTADOS}

$\mathrm{Na}$ busca nas bases de dados, identificaram-se (86) publicações potencialmente elegíveis. Após a leitura do título e resumo de cada publicação, (11) eram duplicadas nas bases de dados e foram excluídas. Do total restante (75), após aplicação dos critérios de seleção, foram excluídas (65) por não serem estudos primários. Assim, a amostra da revisão integrativa foi composta de (10) estudos primários, conforme a figura 1. Ressalta-se que não foram utilizadas outras fontes de publicações, tais como busca manual das referências dos estudos primários incluídos na revisão e literatura cinza. Na presente revisão, foram analisados os 10 artigos que atenderam aos critérios de inclusão previamente estabelecidos e, a seguir, apresenta-se na figura 1 o panorama geral dos artigos elencados na amostra.

Quadro 1 - Caracterização dos estudos segundo código A1 a A10, autores, referência, ano de publicação, periódico, e local de busca. Recife, PE, Brasil, 2020.

\begin{tabular}{|c|c|c|c|c|}
\hline CÓDIGO & $\begin{array}{c}\text { AUTORES } \\
\text { (Referência) }\end{array}$ & ANO & PERIÓDICO & $\begin{array}{c}\text { LOCAL DE } \\
\text { BUSCA }\end{array}$ \\
\hline A1 & $\begin{array}{l}\text { Elena Bohomol, } \\
\text { Eliana Ferreira de } \\
\text { Melo"1. }^{11}\end{array}$ & 2019 & REV. SOBECC & MEDLINE \\
\hline A2 & $\begin{array}{l}\text { Evelyn Alves San- } \\
\text { tos, Aline Natália } \\
\text { Domingues, Aline } \\
\text { Helena Appoloni } \\
\text { Eduardo }^{12} \text {. }\end{array}$ & 2019 & $\begin{array}{l}\text { Revista Enferme- } \\
\text { ría Actual }\end{array}$ & LILACS \\
\hline A3 & $\begin{array}{l}\text { Maria Fernanda } \\
\text { do Prado Tostes, } \\
\text { Cristina Maria } \\
{\text { Galvão }{ }^{13} \text {. }}\end{array}$ & 2019 & $\begin{array}{l}\text { Rev Gaúcha } \\
\text { Enferm }\end{array}$ & LILACS \\
\hline A4 & $\begin{array}{l}\text { Ingrid Moura } \\
\text { de Abreu, Ruth } \\
\text { Cardoso Rocha, } \\
\text { Fernanda Valéria } \\
\text { Silva Dantas } \\
\text { Avelino, David } \\
\text { Bernar Oliveira } \\
\text { Guimarães, } \\
\text { Lidya Tolstenko } \\
\text { Nogueira, Maria } \\
\text { Zélia de Araújo } \\
\text { Madeira }^{14} \text {. }\end{array}$ & 2019 & $\begin{array}{l}\text { Rev Gaúcha } \\
\text { Enferm }\end{array}$ & BDENF \\
\hline
\end{tabular}




\begin{tabular}{|c|c|c|c|c|}
\hline A5 & $\begin{array}{l}\text { Núbia Conceicião } \\
\text { Santos Ferreira, } \\
\text { Luciane Ribiro, } \\
\text { Érica Toledo } \\
\text { Mendoncha, Mari- } \\
\text { lane Oliveira Fani } \\
\text { Amaro' }{ }^{5} \text {. }\end{array}$ & 2019 & $\begin{array}{l}\text { Revista de } \\
\text { Enfermagem do } \\
\text { Centro-Oeste } \\
\text { Mineiro }\end{array}$ & LILACS \\
\hline A6 & $\begin{array}{l}\text { Toti, lan Cesar } \\
\text { Cardoso, Bitten- } \\
\text { court, Jaqueline } \\
\text { Ferreira Ventura, } \\
\text { Borel, Manuela } \\
\text { Gomes Campos, } \\
\text { Monteiro, Thayen- } \\
\text { ne Barrozo Mota, } \\
\text { Silva, Camila } \\
\text { do Nascimento, } \\
\text { Thofehrn, Maira } \\
\text { Buss'16. }\end{array}$ & 2020 & $\begin{array}{l}\text { Journal of nursing } \\
\text { health }\end{array}$ & BDENF \\
\hline A7 & $\begin{array}{l}\text { Cátia Cristiane } \\
\text { Matte Dezordi, } \\
\text { Eniva Miladi Fer- } \\
\text { nandes Stumm }{ }^{17} \text {. }\end{array}$ & 2018 & $\begin{array}{l}\text { J Nursing UFPE } \\
\text { on line }\end{array}$ & BDENF \\
\hline A8 & $\begin{array}{l}\text { Fujii Neta, A, } \\
\text { Tonini, N. S., Luz, } \\
\text { M. S. D, Martins, } \\
\text { L. K., Oliveira, R. } \\
\text { P. D, Santos, P. } \\
\text { R. D'18. }\end{array}$ & 2019 & $\begin{array}{l}\text { Nursing (São } \\
\text { Paulo) }\end{array}$ & BDENF \\
\hline A9 & $\begin{array}{l}\text { Rayanne Morais } \\
\text { de Souzza, Maria } \\
\text { Gabriella Silva } \\
\text { Araújo, Regina } \\
\text { Célia Sales San- } \\
\text { tos Verissimo, Isa- } \\
\text { bel Comasssetto, } \\
\text { Fabiana Andrea } \\
\text { Soares Ferreira, } \\
\text { Thais Honório } \\
\text { Lins Bernardo'. }\end{array}$ & 2016 & Rev. SOBECC & LILACS \\
\hline A10 & 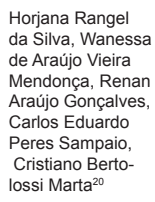 & 2019 & $\begin{array}{l}\text { Revista Enfer- } \\
\text { magem Atual In } \\
\text { Derme }\end{array}$ & BDENF \\
\hline
\end{tabular}

Fonte: Autoria própria, 2020.

Quadro 2 Caracterização dos estudos código A1 a A10 segundo tipo de estudo, áreas e tema do estudo. Recife, PE, Brasil, 2020.

\begin{tabular}{|c|c|c|c|}
\hline CÓDIGO & TIPO DE ESTUDO & ÁREAS DE ATENÇÃO & TITULO DO ESTUDO \\
\hline A1 & Quantitativa & Segurança do paciente & $\begin{array}{l}\text { Cultura De Segurança } \\
\text { Do Paciente Em Centro } \\
\text { Cirúrgico: Percepção } \\
\text { Da Equipe De Enfer- } \\
\text { magem }\end{array}$ \\
\hline A2 & Quantitativa & $\begin{array}{l}\text { Enfermería-periope- } \\
\text { ratoria }\end{array}$ & $\begin{array}{l}\text { Lista de verificación de } \\
\text { seguridad quirúrgica: } \\
\text { conocimientos y desa- } \\
\text { fíos para el equipo del } \\
\text { centro quirúrgico. }\end{array}$ \\
\hline A3 & Estudo transversal & Segurança do paciente & $\begin{array}{l}\text { Lista de verificação de } \\
\text { segurança cirúrgica: be- } \\
\text { nefícios, facilitadores e } \\
\text { barreiras na perspectiva } \\
\text { da enfermagem }\end{array}$ \\
\hline A4 & $\begin{array}{l}\text { Estudo transversal e } \\
\text { analítico }\end{array}$ & Segurança do paciente & $\begin{array}{l}\text { Cultura de segurança } \\
\text { do paciente em centro } \\
\text { cirúrgico: visão da } \\
\text { enfermagem }\end{array}$ \\
\hline A5 & Qualitativa & Segurança do paciente & $\begin{array}{l}\text { Checklist De Cirurgia } \\
\text { Segura: Conhecimento } \\
\text { E Utilização Do Instru- } \\
\text { mento Na Perspectiva } \\
\text { Dos Técnicos De Enfer- } \\
\text { magem }\end{array}$ \\
\hline A6 & Qualitativo & Segurança do paciente & $\begin{array}{l}\text { Percepções dos } \\
\text { profissionais de enfer- } \\
\text { magem na aplicação } \\
\text { do checklist de cirurgia } \\
\text { segura }\end{array}$ \\
\hline A7 & Quantitativa & $\begin{array}{l}\text { Enfermagem Perio- } \\
\text { peratória }\end{array}$ & $\begin{array}{l}\text { Security Attitudes Of A } \\
\text { Team Before And After } \\
\text { Safe Surgery Checklist } \\
\text { Implementation }\end{array}$ \\
\hline
\end{tabular}

\begin{tabular}{|c|l|l|l|}
\hline A8 & Quantitativa & Segurança do paciente & $\begin{array}{l}\text { Segurança do paciente } \\
\text { e cirurgia segura: taxa } \\
\text { de adesão ao checklist } \\
\text { de cirurgia segura em } \\
\text { um hospital escola }\end{array}$ \\
\hline A9 & Quantitative & Patient safety & $\begin{array}{l}\text { Safe surgery checklist } \\
\text { applicability in hospital } \\
\text { surgery centers }\end{array}$ \\
\hline A10 & Qualitativa & Segurança do Paciente & $\begin{array}{l}\text { Percepção da equipe } \\
\text { de enfermagem quanto } \\
\text { as } \\
\text { contribuições da } \\
\text { utilização do checklist } \\
\text { de cirurgia segura }\end{array}$ \\
\hline
\end{tabular}

Fonte: Autoria própria, 2020.

\section{DISCUSSÃO}

Estabelecimento da comunicação e interação entre a equipe

A comunicação é essencial nas interações humanas, podendo ser utilizada na atribuição de sentido. Assim, se constitui um poderoso instrumento básico no processo do cuidar, viabilizando a construção de um relacionamento efetivo com o paciente e os membros da equipe. Possibilita a melhor compreensão das necessidades do paciente, família e comunidade, pois envolve trocas e compartilhamento verbais e não verbais, abrangendo os sentimentos e emoções que as pessoas podem transmitir em um relacionamento interpessoal. Não há interação sem comunicação, portanto ambas são inerentes ao cuidado, sendo habilidades necessárias para que o cuidado se efetive ${ }^{9}$.

A comunicação segura e efetiva entre a equipe de saúde também é um fator determinante para reduzir riscos, a partir da valorização da percepção, de atitudes e comportamentos de todos os profissionais envolvidos no cuidado ao paciente, com vistas à promoção da cultura de segurança, estando inserida no item 2 das metas internacionais da segurança do paciente. Para isso, a equipe precisa estar encorajada e comprometida individual e coletivamente, de modo a compreender a importância e a necessidade do uso da lista de verificação do checklist e, posteriormente, atribuir ações corretivas por meio de indicadores seguros ${ }^{10}$.

A utilização do checklist cirúrgico como parte do processo de trabalho ratifica a centralidade do cuidado no paciente, sendo uma ferramenta com potencial para a qualificação da assistência. Acredita-se que a implantação de protocolos, como o do checklist nos serviços promova a comunicação efetiva entre os profissionais da equipe multiprofissional ${ }^{5}$. Para que ocorra uma interação entre a equipe multidisciplinar é preciso o compartilhamento e troca de saberes e experiências, para que ocorra um crescimento na relação interpessoal. A consciência de que as dificuldades de relacionamento 
e os conflitos são reais nas relações faz-se necessária para que haja superação e o entendimento dos conflitos para interação e a união da equipe ${ }^{9}$.

Um dos aspectos que pode diminuir o número de erros é a comunicação de equipe multiprofissional, quando a mesma é efetiva, o máximo de informações, que são registradas, será repassado ao outro profissional que também estará prestando assistência. Os autores destacam ainda como principal vantagem para os profissionais de enfermagem diz respeito à fomentação que esta ferramenta contribui para tomada de decisões e atitudes que buscam a segurança do paciente e da equipe, evitando falhas, proporcionando ações efetivas, a fim de evitar erros relacionados à cirurgia ${ }^{11}$.

A comunicação pode ser um fator motivacional para os profissionais de enfermagem que trabalham em equipe, e se há interação efetiva evitam-se sentimentos de desprezo, insegurança, medo, tristeza, opressão e insatisfação. Logo se evidencia a importância de estreitar, estimular e fortalecer as relações ${ }^{9}$. Observa-se desta forma que emerge a necessidade deste diferencial na assistência em saúde, na busca evidente em estabelecer padrões de segurança, que é possibilitado pelo método sistematizado (checklist) no intuito de prevenir erros e falhas humanas.

Além disso, a complexidade do cenário cirúrgico deve ser considerada, uma vez que a tecnologia e os recursos materiais se articulam com altas cargas de estresse. Não só a habilidade técnica é o suficiente, mas a capacidade de se comunicar de forma eficaz, reconhecendo limitações, aprender com os erros, trabalhar em equipe para garantir a qualidade de uma assistência segura ${ }^{7}$.

\section{Cuidado seguro para redução do medo e da ansie- dade.}

A interação da equipe multiprofissional com o paciente e família traduzem-se em elementos que convergem para segurança do paciente. $\mathrm{O}$ trabalho em equipe gera construção coletiva de ações e contribui para que as dificuldades mesmo estando presentes, poderão ser superadas a partir da troca de informações e pela construção de planos terapêuticos de cunho interdisciplinares $^{3}$.

Quando a informação é quebrada ou modificada, pode haver uma piora na comunicação, o que interfere no processo de trabalho e na qualidade da assistência. Por isso é importante o estreitamento dos laços de co- municação entre profissionais, clientes e a família, para que haja um fortalecimento das relações estabelecidas, permitindo que as equipes trabalhem de forma satisfatória, criando estratégias que melhore a comunicação e o trabalho em equipe ${ }^{9}$.

Nas relações do cuidado, a comunicação precisa possuir todos os elementos do processo, é entender e ser entendido por meio do compartilhamento e interação, para que haja efetividade na relação, pois em algumas situações pode haver atritos que interferem diretamente nas ações do cuidado e, posteriormente, em todo processo terapêutico. A comunicação efetiva quando estabelecida no processo reflete uma relação de confiança reduzindo o medo e a ansiedade ${ }^{9}$.

A visita pré-operatória é uma estratégia para a humanização do cuidado de enfermagem, tendo como objetivo instrumentalizar o usuário com informações, logo reduz sua ansiedade angústia e medo do procedimento cirúrgico. A avaliação emocional do paciente deve ser realizada pelo profissional de saúde à medida que também são colhidas outras informações relevantes, tais como história anestésica, alergias, entre outras. O profissional também promove educação em saúde, preparando o paciente para a cirurgia e recuperação anestésica de forma individualizada, com linguagem que possa atingir o seu entendimento e a necessidade de aprendizado do individuo ${ }^{12}$.

Estudo realizado em 2019 em um hospital universitário sobre as contribuições das ações educativas no período pré-operatório apontou que as maiores contribuições foram a tranquilidade, segurança, autonomia e o auxílio no entendimento a ser vivenciado. Após o estudo, os pacientes relataram que ficaram menos ansiosos, com o melhor entendimento de seus processos cirúrgicos. Quando questionados sobre os sentimentos, relataram o medo, nervosismo, preocupação e tristeza. Logo, se percebe uma relação com a incerteza dos eventos e os possíveis riscos relacionados ao procedimento cirúrgico ${ }^{13}$.

Estudo realizado no ano de 2015 com crianças mostrou que fornecimento de matérias pré-operatório contendo informações educativas, reduziram as preocupações das crianças e dos seus pais, quando comparadas as que não tiveram nenhum preparo ${ }^{14}$. Outro aspecto a ser considerado no ambiente cirúrgico é a espiritualidade. Participantes de um estudo, afirmaram que a fé foi uma grande aliada, deu suporte para o enfrentamento das situações evidenciadas no período perioperatório, diminuindo angústias e medo, já que aspectos 
espirituais mantêm relação direta com os sintomas psicossomáticos que envolvem as doenças e procedimentos cirúrgicos. Assim, a espiritualidade atua de forma benéfica nos sintomas da ansiedade, angústias depressão e dores ${ }^{15}$.

As orientações e o apoio psicológico são considerados benéficos no período que antecede a um procedimento cirúrgico, pois à medida que o paciente esclarece suas dúvidas, diminui seus medos, gerando mecanismos eficazes para melhor o enfrentamento dos momentos a ser vivenciados, gerando uma melhor compreensão que contribui para redução de sua ansiedade ${ }^{13}$.

O uso do checklist aplicado na prática pode ser um facilitador das interações, quando o paciente e sua família são orientados sobre o que acontecer no período pré-operatório. Para tanto, também se considera a necessidade em possuir um local para registrar essas ações, possibilitando a tomada de decisões em equipe ${ }^{16}$.

\section{Conhecimento do protocolo de cirurgia segura salva vidas}

O protocolo construído com base no desafio global da OMS tem o objetivo em aumentar os padrões de qualidade da assistência cirúrgica mundial. Assim, o checklist de segurança cirúrgica é considerado um elemento chave para a redução de eventos adversos. A Organização Mundial da Saúde instituiu uma lista básica, podendo ser adaptada e modificada conforme as necessidades do serviço prestado. Além de contribuir individualmente com a segurança do paciente, compreende-se uma base de dados montada possibilitando decisões gerais de melhorias do processo de trabalho ${ }^{2-7}$.

Ao elaborar e avaliar um modelo de checklist nos períodos pré e pós-operatório, construído de forma participativa e dialógica com profissionais atuantes em suas próprias unidades cirúrgicas, possibilita-se trazer a realidade as necessidades, poder de decisão e melhor resolução. Esta ação também permite aos pesquisadores e profissionais envolvidos na construção deste instrumento, a disponibilidade e interesse em inovar de forma simples e prática as ações do cuidado. Existe a evidência de um enriquecimento teórico, gerencial e prático, permitindo a possibilidade de mensuração ao considerar o uso deste instrumento um aliado na melhoria da qualidade da assistência.

Desta forma, a ação requer dos profissionais mudanças no comportamento, contínua busca pelo conhecimento e sua disseminação, além do desenvolvimento de novas habilidades e modificação de atitudes. É importante destacar que o impacto da lista de verificação é susceptível ao não sucesso, pois dependerá do processo de implantação de cada hospital, fatores organizacionais e culturais ${ }^{17-21}$.

Neste contexto, na área de assistência em saúde deve considerar sua variabilidade, ou seja, cada paciente necessita de recursos estruturais, matérias e profissionais prontos a identificar e atender as suas necessidades individuais. Cada instituição tem sua própria realidade, devendo assim estabelecer critérios, normas e ambiente que favoreça uma assistência individualizada, adequando-se a cada procedimento, podendo ser realizadas modificações e adaptações ${ }^{7-22}$.

Ao nível organizacional, as instituições de saúde devem buscar como exemplos de modelos de gestão de risco, usadas por Organizações de Alta Confiabilidade (OAC), conhecidas tradicionalmente, por gerenciar com eficiência altos riscos dos processos de trabalho em áreas complexas e perigosas, visando mitigar os riscos de incidentes e eventos adversos. Alguns aspectos a ser considerado é como lidam com erros: antecipação e consciência de riscos; capacidade de aprender com os erros; relutância em simplificar; envolvimento de todos nas práticas de segurança; resiliência ou redesenhar sempre o sistema ${ }^{5-23}$.

Outro aspecto a ser considerado é o papel dos líderes ou supervisores nas pontas dos serviços, que deveriam orientar seus padrões de comportamento de liderança, para que haja uma busca pela promoção da cultura de segurança, que engloba e valorizam as motivações, atitudes, percepções e os comportamentos que determinam o compromisso com a segurança do paciente. $O$ fator humano é também considerado um fator determinante para a cultura de segurança nas OAC, a exemplo do centro cirúrgico, o alto nível gradientes de autoridade e a hierarquia elevada, que muitas vezes compromete a comunicação entre os profissionais da equipe cirúrgica aumentando o risco de incidentes ${ }^{5-24}$.

Uma pesquisa realizada na França sobre a implantação da lista de verificação obteve como resultado a falta de informação da equipe. Na percepção desta pesquisa, a lista de verificação, constitui-se mais um documento administrativo que serve apenas de complemento, do que algo útil para a segurança do paciente. Reafirmando esta pesquisa, autores advertem sobre a deficiente formação relacionada ao tema, falta de formação específica em gerenciamento de risco, gestão inadequada do conhecimento e escassez de pesquisas 
e evidências sobre o assunto, pois a falta de material bibliográfico e literário acarreta em pouco conhecimento $^{17-21}$.

A cultura de segurança é uma construção diária vinculada a recursos administrativos, que requer uma transição de uma cultura de silêncio e de punição para uma cultura de mudança, onde o objetivo é uma assistência segura. Daí um aspecto a ser observado é a criação de políticas de educação permanente nas instituições de saúde ${ }^{1}$. A educação em saúde e a supervisão permanente dos profissionais são fundamentais para o crescimento pessoal e profissional. Assim, implantar ações que contribuam para a cultura de segurança nas instituições de saúde se revertem em melhoria dos indicadores de qualidade ${ }^{18-22}$.

A educação permanente é um instrumento de gestão do cuidado de enfermagem, pois possibilita identificar potências dificuldade inerente ao cotidiano da profissão, bem como possibilita a comunicação, escuta, troca de saberes e formulação de novas ideias, conduzindo toda equipe à compreensão e ao entendimento. Nesse contexto, a segurança do paciente deve ser monitorada e mensurada de forma clara e precisa nos serviços de saúde ${ }^{10-15}$

Os registros escritos contribuem para a qualidade da assistência, sendo também importantes indicadores de processos e resultados. Nas instituições de saúde brasileiras, o NSP é a responsável pela análise dos registros de incidentes notificados, possibilitando a avaliação, revelando as condições sobre as quais os serviços de saúde são prestados e direcionando as práticas seguras $^{19-20}$.

\section{CONCLUSÃO}

Apesar da atual preocupação envolvendo a segurança do paciente, percebe-se que a implementação de metas internacionais ainda é um desafio em muitos serviços, pois demanda uma mudança para uma cultura de segurança. Alguns profissionais de saúde ainda são resistentes na mudança de suas práticas e acreditam que metas de segurança sejam mais uma tarefa burocrática.

Portanto, reconhece-se que a segurança do paciente deve ser respeitada em todas as áreas, tornando esta meta um grande desafio, sobretudo no Centro Cirúrgico em que o paciente se torna indefeso e vulnerável, principalmente diante do ato cirúrgico. Neste sentido, verifica-se que o checklist favorece a comunicação entre a equipe devendo incluir o paciente e seus fami- liares.

A participação do enfermeiro é essencial neste processo, bem como da realização da sistematização da assistência de enfermagem. É imprescindível realizar ações de educação em saúde e sensibilizar a equipe cirúrgica que o agir com segurança salva vidas. As instituições devem implementar um programa de segurança e contribuir para que as metas sejam cumpridas e os eventos adversos sejam notificados.

\section{REFERÊNCIAS}

1. Santos JS, Souza DO, Morais AC, Santana CLM, Rodrigues US, Rodrigues EP. Test check pilot list of safe surgery: experience report. REUFPI. 2017;6(1):76-9.

2. Organização Mundial da Saúde. Segundo desafio global para segurança do paciente. Manual - Cirurgias Seguras Salvam Vidas: (Orientações para cirurgias seguras da OMS). Rio de Janeiro; 2009.

3. Reis AT et al. O significado da segurança do paciente cirúrgico pediátrico para a equipe de enfermagem. Cogitare Enfermagem. 2016;21(1):01-8.

4. Brasil, Agência Nacional de Vigilância Sanitária. RDC n36 de 25 de julho 2013. Institui ações para segurança de pacientes em serviços de saúde e de outras providências. Brasil, 2013.

5. Gomes CDPP, et al. Percepção de uma equipe de enfermagem sobre a utilização do checklist no centro cirúrgico. Revista SOBECC. 2016;21(3):140-45.

6. Oliveira $\mathrm{MCB}$, et al. Adesão do cheklist cirúrgico à luz da Cultura de segurança do paciente. Revista SOBECC. 2018;23(1):36-42.

7. Alpendre, et al. Cirurgia segura: Validação de checklit pré e pós- operatório. Revista Latino-americana de enfermagem. 2017;25:e2907.

8. Bock A, et al. Ações educativas no período perioperatório em um hospital universitário: Percepção de pacientes cirúrgicos. Revista de enfermagem UFSM. 2019;9(28):2-20.

9. Santos CMDC, Pimenta CADM, Nobre MRC. A estratégia PICO para a construção da pergunta de pesquisa 
e busca de evidências. Revista Latino-Americana de Enfermagem. 2007;15(3): 508-11.

10. Oliveira JLCD, Magalhães AMMD, Misue ML. Métodos mistos na pesquisa em enfermagem: possibilidades de aplicação à luz de Creswell. Texto \& Contexto-Enfermagem. 2018;27(2):1-10.

11. Bohomol E, Melo EF. Cultura de segurança do paciente em centro cirúrgico: percepção da equipe de enfermagem. Revista SOBECC. 2019;24(3):132-38.

12. Santos EA, Domingues NA, Eduardo AHA. Lista de verificação para segurança cirúrgica: conhecimento e desafios para a equipe do centro cirúrgico. Enfermería Actual de Costa Rica. 2019;38:75-88.

13. Tostes MFP, Galvão CM. Lista de verificação de segurança cirúrgica: benefícios, facilitadores e barreiras na perspectiva da enfermagem. Revista Gaúcha de Enfermagem. 2020;40(SPE).

14. Abreu IM, et al. Cultura de segurança do paciente em centro cirúrgico: visão da enfermagem. Revista Gaúcha de Enfermagem. 2019; 40(SPE).

15. Ferreira NCS, et al. Checklist de cirurgia segura: conhecimento e utilização do instrumento na perspectiva dos técnicos de enfermagem. Revista de Enfermagem do Centro-Oeste Mineiro. 2019;9.

16. Toti ICC, et al. Percepções dos profissionais de enfermagem na aplicação do checklist de cirurgia segura. Health. 2020;10(1):e20101010.

17. Matte DCC, Fernandes SEM. Atitudes De Segurança De Uma Equipe Antes E Após A Implantação Do Checklist De Cirurgia Segura. Journal of Nursing UFPE/Revista de Enfermagem UFPE. 2018;12(3).

18. Fujii NA, et al. Segurança do paciente e cirurgia segura: taxa de adesão ao checklist de cirurgia segura em um hospital escola. Nursing (Säo Paulo). 2019;33803383.

19. Souza RM, et al. Aplicabilidade do checklist de cirurgia segura em centros cirúrgicos hospitalares. Revista SOBECC. 2016;21(4):192-97.
20. Sampaio CEP. Percepção da equipe de enfermagem quanto as contribuições da utilização do checklist de cirurgia segura. revista enfermagem atual in derme. 2019;87(25).

21. Giannattasio MB, Taniguchi FP. Avaliação da segurança do paciente em cirurgia cardíaca de um hospital Público. Revista SOBECC. 2016;21(3):125-31.

22. Silva TCV, Mazzi NR. A espiritualidade no cuidado perioperatório: a perspectiva do paciente. J. nurs. health. 2019;9(2):e199205.

23. Silva FAA, Silva AGM. Equipe de enfermagem em cirurgia segura: desafios para adesão ao protocolo. Revista enfermagem da UFPI. 2017;6(2):23-9.

24. Silva CSO, et al. Checklist de cirurgia segura: Os desafios da implantação e adesão nas instituições hospitalares brasileiras. Revista espaço ciência e saúde. 2017;5(2):72-86. 